\title{
Electrical properties of electrochemically doped organic semiconductors using light-emitting electrochemical cells
}

\author{
G. Gozzi $^{1,2}$ • L. D. Cagnani ${ }^{2}$ R. M. Faria ${ }^{2}$ - L. F. Santos ${ }^{1,3}$
}

Received: 11 December 2015 /Revised: 6 April 2016 / Accepted: 13 April 2016/Published online: 20 April 2016

(C) Springer-Verlag Berlin Heidelberg 2016

\begin{abstract}
We present a study of the electrical properties of electrochemically doped conjugated polymers using polymeric light-emitting electrochemical cells (PLECs) and interpreting the results according to a phenomenological model (PM) which assumes that, above the device turn-on voltage, the bulk transport properties of the doped organic semiconductor are responsible for the main contribution to the whole device conductivity. To confirm the predictions of this model, the dependence of the conductivity of PLECs with different parameters is evaluated and compared with the behavior expected for a doped semiconducting polymeric material. The organic semiconductor doping level, the blend concentration of organic semiconducting molecules, the device thickness, the charge carrier mobility, and the temperature are the parameters varied to perform this analysis. We observed that the device conductivity is independent of the active layer thickness, weakly dependent on the temperature, but strongly dependent on the semiconductor doping level, on the semiconductor fraction in the blend, and on the intrinsic charge carrier mobility. These results were well described by the variable range hopping (VRH) model, which has been widely employed to describe the charge transport in doped
\end{abstract}

G. Gozzi

giovanigozzi@rc.unesp.br

1 Departamento de Física, Instituto de Geociências e Ciências Exatas, Universidade Estadual Paulista - UNESP, Rio Claro 13506-900, Brazil

2 Instituto de Física de São Carlos, Universidade de São Paulo, São Carlos 13566-590, Brazil

3 Departamento de Física, Instituto de Biociências, Letras e Ciências Exatas, Universidade Estadual Paulista - UNESP, São José do Rio Preto 15054-000, Brazil semiconducting polymeric materials, confirming the prediction of the phenomenological model. The current analysis demonstrates that PLECs are a suitable system for studying, in situ, the electrochemical doping of semiconducting polymers, permitting the evaluation of material properties as, for instance, the density of electronic charge carriers (and, consequently, the ionic charge carrier concentration) necessary to achieve the maximum electrochemical doping level of the organic semiconductor.

Keywords Organic electronics $\cdot$ Light-emitting electrochemical cells $\cdot$ Semiconducting polymers . Electrochemical doping $\cdot$ Electrical properties

\section{Introduction}

Polymeric light-emitting electrochemical cells (PLECs) consist of a thin polymer blend film, comprising a semiconducting polymer, an ion transport polymer, and an ionic salt, sandwiched between two electrodes [1]. This class of devices commonly exhibits a low turn-on voltage (approximately equal to the semiconductor energetic bandgap, $V_{\mathrm{on}} \approx E_{\mathrm{g}} / e$ ), irrespective of the work function of the conductive materials used as electrodes, as a consequence of the electrochemical doping of the conjugated polymer produced by the ionic separation in the solid electrolyte phase of the polymer blend under an external electric field $[1,2]$. Such effect occurs due to the blend morphology, which constitutes a 3-D interpenetrating network, formed by the solid electrolyte (usually a mixture of polyethylene oxide, PEO, and a lithium salt), permitting both ionic and electronic charge transport through the active layer. For several years, the technological potential of PLECs was not totally exploited due to few, but relevant, drawbacks as, for instance, the low operation lifetime and 
poor chemical stability. However, recent works reporting the achievement of stable and long-lasting PLECs have increased the interest on the investigation of the electrical properties and on the understanding of the operation mechanisms of this class of devices [3-8]. The development of PLECs is also motivated by the ease of process and by the low manufacturing cost, permitting the production of devices with totally organic electrodes $[9,10]$ and the use of innovative deposition techniques as slot die [11] and spray coating [12]. In the subject of the fundamental properties of the device operation, several studies have been carried out focusing on the electronic and ionic charge transport properties, on the electric field distribution during a steady-state operation regime, and on the electrochemical doping of the semiconducting material. In particular, the dynamics of the electrochemical doping during the device operation is a topic that was not thoroughly studied and still remains unclear.

When a relatively low d.c. bias (below $1 \mathrm{~V}$ ) is applied to the electrodes of a PLEC, the electric field in the active layer promotes the salt dissociation and the ion transport in the solid electrolyte phase. Considering that the electrodes are blocking for ions, the ionic species continuously accumulate near the electrodes until the electric field in the bulk becomes negligible. At this condition, intense electric fields at the metal/active layer interfaces are established, and electronic charge carriers can overcome the energy barrier, being injected into the organic semiconductor phase. The model that describes this phenomenon is known as ionic space-charge-assisted current injection [13]. Increasing the bias voltage until the semiconductor bandgap $\left(E_{\mathrm{g}} / e\right)$, the injected electronic carriers become numerous enough to counterbalance the amount of ionic charges in the solid electrolyte and the electrochemical doping of the semiconducting polymer occurs [14]. In this situation, a $p$-doped region is formed in the proximity of the anode whereas an $n$-doped region is formed near the cathode, with a very thin (about less than $10 \%$ of the total layer thickness) insulating region (non-doped) separating the doped regions $[15,16]$. When this $p$ - $i$ - $n$ junction ( $p$-doped, insulating, $n$-doped) is established, the electric field is predominantly concentrated in the insulating region [14-16], as well as the electronic charge carrier recombination and the emission of light.

The ionic space-charge-assisted current injection and the $p$ $i-n$ junction models were considered conflicting models until 2010, when a unified model was proposed to explain the operation mechanism of PLECs as a whole [17]. This model considers that the electrochemical doping of the semiconducting polymer doping depends on the electronic charge carrier injection, which is promoted by the ionic space-chargeassisted mechanism. Additionally, the model predicts that, even after the $p-i-n$ junction formation, the electric field at the metal/polymer interfaces still remains, in addition to the electric field in the insulating layer, which was corroborated by experimental results $[17,18]$. The explication for such behavior is that the voltage confined at the interfaces, $V_{\text {in }}$, is necessary to maintain the electric current constant along the entire device. More recently, a phenomenological model (PM) [19] was proposed, considering the unified model and the influence of the electric resistance of the doped layers, to explain the operation of PLECs in transient [20] and alternated current (a.c.) [21] conditions. The generality of the PM permits to successfully describe the electrical properties of PLECs in a broad range of applied voltage (below and above the turn-on voltage), for d.c., transient, and a.c. regimes. The main idea behind this model is that the device electrical behavior is described in terms of an apparent conductivity, which is obtained by considering the device as a uniform material layer, with the total equivalent electric resistance normalized by the geometric parameters (film thickness and electrode area) of the device [19].

In a previous work [19], the authors proposed a phenomenological model (PM) based on experiments performed in PLECs with different thicknesses and with different semiconducting polymers, assuming that, for voltages much higher than the PLEC turn-on voltage $\left(V_{\text {on }}\right)$, the PLEC "conductivity" is the same of the doped semiconductor. In order to confirm this assumption in a more general way, we present in the current work a study of the dependence of the conductivity of PLECs on additional parameters: organic semiconductor doping level, organic semiconducting polymer concentration, and temperature. Moreover, to guarantee operation under the high-voltage electrochemical doping regime, all the experiments were carried out with the devices operating at voltages much higher than the device turn-on voltage (above $5 \mathrm{~V}$ ). As general results, we show that the PLEC conductivity depends on these parameters according to a variable range hopping (VRH) model, which has been widely applied to describe the charge transport in doped semiconducting polymers, confirming the PM predictions.

\section{Experimental procedures and materials}

\section{Materials}

The active layer of the devices was composed of the mixture of a semiconducting conjugated polymer and a solid polymeric electrolyte. The polyfluorene derivative poly $[(9,9-$ dioctylfluorenyl-2,7-diyl)-co-(1,4-vinylenephenylene)] (green-emitting polymer, ADS_GE) was used as the organic semiconductor (OS) in most of the experiments. In order to evaluate the influence of the charge carrier mobility in the polymeric electrolyte, some devices were also fabricated using poly[(9,9-dioctylfluorene-2,7-diyl)] (blue-emitting polymer, ADS_BE) as a semiconducting polymer. Both organic semiconductors were purchased from American Dye Source Inc. (ADS). The polymeric electrolyte was produced 
by mixing poly(ethylene oxide), $\mathrm{PEO}$, (Aldrich, $\mathrm{Mw}=5 \times 10^{6} \mathrm{~mol} /$ g) and lithium trifluoromethanesulfonate, $\mathrm{CF}_{3} \mathrm{SO}_{3} \mathrm{Li}$, (LiTri, Aldrich), at different concentrations of the lithium salt.

\section{Sample fabrication}

Sample preparation was carried out completely inside a glove box (Inertec) under nitrogen atmosphere, with moisture and oxygen concentration below $10 \mathrm{ppm}$. Solutions of the different active materials were spin coated onto ITO-covered glass substrates and baked at $120{ }^{\circ} \mathrm{C}$ for $15 \mathrm{~min}$, to guarantee total solvent evaporation. Metallic aluminum top electrodes (150 $\mathrm{nm}$ thick) were deposited by thermal evaporation, in a high-vacuum deposition system, through mechanical shadow masks, comprising an effective area of $10 \mathrm{~mm}^{2}$. Different film thicknesses were achieved by controlling the spin-coating rotation speed from 500 up to $3000 \mathrm{rpm}$, using the same solution concentration. Different blend compositions were produced by mixing different amounts of base solutions to obtain a desired mass ratio of the components (OS/PEO/LiTri). The base solutions of the conjugated polymers and PEO were prepared in chloroform at 15 and $10 \mathrm{mg} / \mathrm{mL}$, respectively. LiTri base solution was prepared in acetonitrile at $30 \mathrm{mg} / \mathrm{mL}$. Devices produced using different semiconducting polymers and different layer thicknesses were produced by using blends at the same mass ratio $(1: 1: 0.1)$. Samples produced with different salt concentrations $(C)$ were produced with a OS/PEO mass ratio of 1:1, whereas samples produced with different semiconductor concentrations used the same $\mathrm{PEO} /$ salt mass ratio (1:0.4) and a OS/PEO mass ratio given by $X:(1-X)$, where $X$ represents the OS relative concentration.

\section{Electric measurements}

Current-voltage $(I-V)$ measurements were performed with a Keithley source/meter unit model 2400 . The maximum voltage variation rate was $500 \mathrm{mV} / \mathrm{s}$, with a time delay of $1 \mathrm{~s}$ between adjacent points, in order to guarantee the device operation a steady-state condition. Device characterization was carried out keeping the samples inside a low-pressure chamber filled with nitrogen at 1 mbar. Before the measurements, five purge cycles of $\mathrm{N}_{2} /$ vacuum were performed. Device light emission was characterized by luminance-voltage $(L-V)$ measurements using the 2400 source/meter unit and a calibrated photodiode connected to a Keithley electrometer, model 617. The conversion of the photodiode photocurrent to luminance was performed considering the photodiode spectral responsivity, the device electroluminescence (EL) spectrum, and the CIE human eye spectral responsivity [22]. Temperature variation during the electrical measurements was performed within a Janis closed-circuit helium cryostat, controlled by a Scientific Instruments Inc. temperature controller model 9160. The thickness of the device active layer was determined using a Veeco Dektak profilometer model 150.

\section{Results and discussion}

Current-voltage $(I-V)$ and luminance-voltage $(L-V)$ curves for a 190-nm thick PLEC with 1:1:0.1 (OS/PEO/LiTri) blend composition, using ADS_GE as an organic semiconductor, are presented in Fig. 1a. The $I-V$ curve exhibits two linear regions, corresponding to two different transport regimes, where the conductivity is nearly constant, as depicted on Fig. 1b. The PLEC apparent conductivity vs. voltage curve was obtained from the direct differentiation of the $I-V$ curve and considering the device geometrical parameters (active layer thickness and active area). The turn-on voltage $\left(V_{\text {on }}\right)$ of the device is determined from the onset in $L-V$ curve, which is approximately $4.5 \mathrm{~V}$. For voltages right above $V_{\text {on }}$, the conductivity increases exponentially (Fig. 1b), outlining the transition between a low
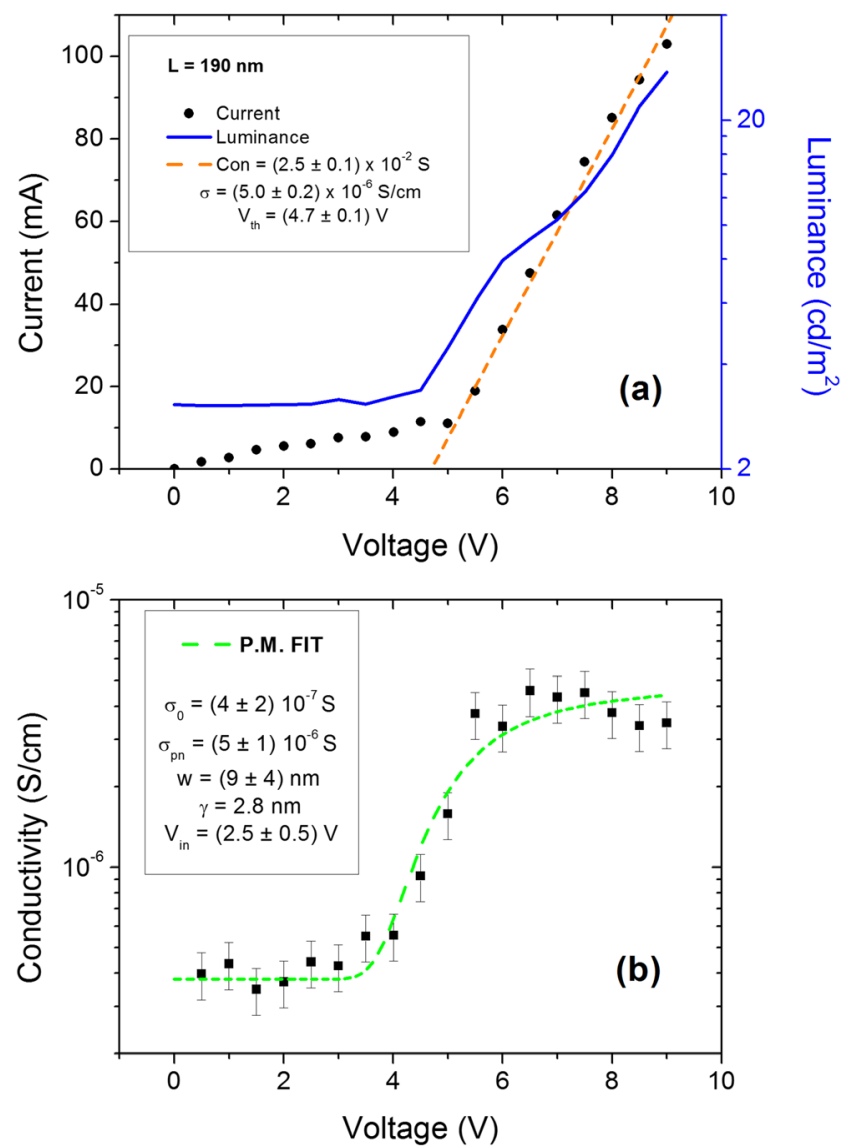

Fig. 1 a Current-voltage $(I-V)$ and luminance-voltage $(L-V)$ characteristic curves of a 190-nm-thick PLEC; b device apparent conductivity vs. voltage curve obtained from the derivative of the $I-V$ and the corresponding fitting by using Eq. 1 
(nearly constant)-conductivity regime to a high-conductivity regime, which also becomes nearly constant.

In the low-conductivity regime, the steady-state current is governed by the injection of electronic charge carriers from the electrodes, assisted by the accumulated ionic space charges next to the metal/polymer interfaces, resulting, according to the PM [19], in an apparent conductivity $\sigma_{0}$ for the whole device. The steep increase on the conductivity around $V_{\text {on }}$ is a consequence of the tunneling of the electronic charge carriers from the doped layers into the insulating layer of the $p-i-n$ junction. In this regime, the charge carrier recombination becomes high enough to promote a visible light emission and the device turn-on voltage is reached. The exponential growth of the apparent conductivity, $\sigma$, depends directly on the thickness of the insulating layer, $w$, and on the electronic localization radius, $\gamma$. In the high-voltage regime, when the apparent conductivity becomes again nearly constant, the device whole conductivity is limited by the conductivity of the electrochemically doped layers, $\sigma_{\mathrm{pn}}$, considering the assumption that both layers ( $p$-doped and $n$-doped) have the same conductivity. Moreover, the thickness of the insulating layer of the $p-i-n$ junction decreases drastically, becoming much thinner than the whole device active layer thickness, which makes its contribution to the device resistance negligible. Such a situation leads to two approximations: (i) the thickness of the two electrochemically doped layers (ECDLs) is about the same of the whole active material and (ii) the whole device apparent conductivity is nearly the same of the ECDLs.

The apparent conductivity of the PLEC shown in Fig. 1b was fitted by using the expression for the apparent conductivity of a PLEC defined in ref. [19]:

$\sigma(V)=\frac{\sigma_{\mathrm{pn}}}{1+\frac{w}{L} \frac{\sigma_{\mathrm{pn}}}{\sigma_{i}}}=\frac{\sigma_{\mathrm{pn}}}{1+\frac{w}{L} \exp \left(\frac{w}{\gamma} \frac{E_{\mathrm{g}}}{V}\right)}$

where $\sigma_{\mathrm{pn}}$ represents the conductivity of the ECDLs, $\sigma_{\mathrm{i}}$ the conductivity of the non-doped (intrinsic) semiconductor, $w$ represents the thickness of the insulating layer of the $p-i-n$ junction, $E_{\mathrm{g}}$ the energetic bandgap of the organic semiconductor, and $\gamma$ represents the electronic localization radius, which was considered as $2.8 \mathrm{~nm}$ in our calculations [19]. The parameters obtained from the fittings (Fig. 1b, inset) are $\sigma_{\mathrm{i}}=(4 \pm 2) \times 10^{-7} \mathrm{~S} / \mathrm{cm}, \sigma_{\mathrm{pn}}=(5 \pm 1) \times 10^{-6} \mathrm{~S} / \mathrm{cm}$, $w=9 \pm 4 \mathrm{~nm}$, and $V_{\text {in }}=2.5 \pm 0.5 \mathrm{~V}$, which are in good agreement with previously obtained results [19]. Figure 2 presents the dependence of conductance of a device (ADS_GE/ $\mathrm{PEO} / \mathrm{LiTri}$ ) on the thickness of the active layer, operating in the high-voltage regime. The conductance varies nearly inversely proportional to the active layer thickness, which is an evidence that the electronic charge transport becomes predominantly a bulk effect and, consequently, that the interfaces play a minor role in the device operation in this regime. The

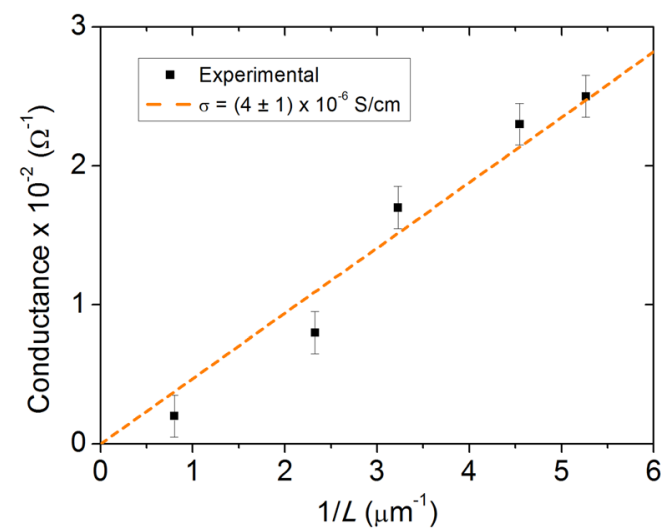

Fig. 2 Dependence of the device conductance on the PLEC active layer thickness

data also reveals that the conductivity of the ECDLs is nearly independent on the device thickness and reaches a value of at about $(4 \pm 1) \times 10^{-6} \mathrm{~S} / \mathrm{cm}$ in the high-voltage regime.

Figure 3 shows that the device apparent conductivity, in the high-voltage regime, is proportional to the organic semiconductor (ADS_GE) concentration in the blend. Such behavior indicates that the density of charge carriers is proportional to the concentration of the organic semiconductor in the blend. Considering that the charge carrier mobility in disordered semiconductors is not strongly affected by doping [23], since the majority charge carrier mobility of intrinsic (non-doped) organic semiconductors can be determined from CELIV measurements (values for ADS_GE and ADS_BE are presented on ref. 19), it is possible to evaluate the density of electronic charge carriers in the organic semiconductor from the conductivity obtained for the ELDL $\left(\sigma_{\mathrm{pn}}\right)$ of PLECs in the highvoltage regime. Using the obtained results of $\sigma_{\mathrm{pn}}$ from PLECs at a constant mass ratio of 1:1:0.1 (OS/PEO/LiTri), we estimate a density of charge carriers of $4.4 \times 10^{17} \mathrm{~cm}^{-3}$ (for ADS_BE) and $5.7 \times 10^{17} \mathrm{~cm}^{-3}$ (for ADS_GE), with quite similar values indicating the proportionality between $\sigma_{\mathrm{pn}}$ and

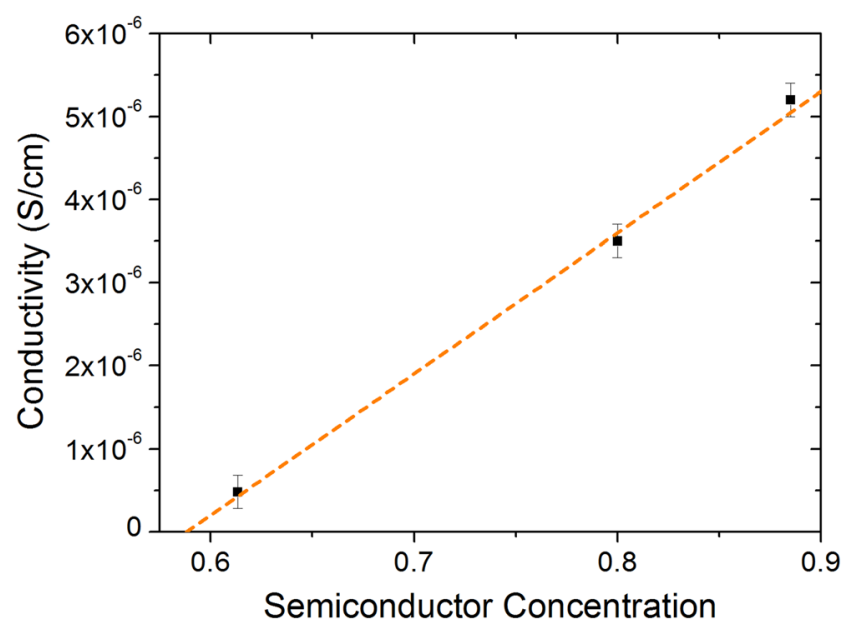

Fig. 3 Conductivity of the PLEC active layer as a function of organic semiconductor concentration 
charge carrier mobility in the semiconducting polymer. However, were employed doped semiconductors to perform the experiment, and the absolute values of the density of charge carriers are in agreement with the typical density of electronic states in intrinsic organic semiconductors [24]. The reason for this discrepancy is that the OS is a component of a blended material, in which the charge carrier mobility may be reduced as a consequence of the insulating phase of the material.

The previous results show that it is reasonable to consider that the active material conductivity is proportional to the semiconductor material concentration, which is intrinsically a $p$-type material, and to the positive charge carrier (holes) mobility. Taking into account that the electronic charge carrier density is proportional to the salt concentration in the blend and that the charge transport in a disordered semiconductor can be modelled by a 3-D Mott variable range hopping process [25], the dependence of the $p$-doped semiconductor polymer conductivity with the dopant salt concentration can be described by the following expression:

$$
\begin{aligned}
\sigma(C) & =\sigma_{0} \exp \left[-\left(\frac{T_{\mathrm{h}}}{T}\right)^{\frac{1}{4}}\right]=\sigma_{0} \exp \left[-\left(\frac{\beta}{T k_{\mathrm{B}} \frac{n_{0}}{\Delta \mathrm{E}} \gamma^{3} C}\right)^{\frac{1}{4}}\right] \\
& =\sigma_{0} \exp \left\{-\left[\frac{\beta \Delta \mathrm{E}}{T k_{\mathrm{B}}}\left(\frac{L_{0}}{\gamma}\right)^{3} \frac{1}{C}\right]^{\frac{1}{4}}\right\}
\end{aligned}
$$

where $\sigma_{0}$ represents the maximum value of the conductivity of the semiconductor that can be achieved in the d.c. regime, $T_{\mathrm{h}}$ the Mott characteristic hopping temperature, and $T$ the experimental temperature. The parameter $\beta$ represents the Mott model dimensional factor (10.3 for 3-D hopping), $k_{\mathrm{B}}$ the Boltzmann constant, $n_{0}$ the density of states at maximum doping $(C=1)$, and $\Delta E$ the energy interval which comprises the density of states. The parameter $L_{0}$ represents the minimum hopping site distance, $\gamma$, the electronic charge carrier localization radius, and $C$ represents the salt concentration in the blend. Figure 4 shows the dependence of the conductivity of the ECDLs on the salt concentration (or doping level) obtained for PLECs produced with ADS_GE as a semiconductor. The experimental data from Fig. $\overline{4}$, fitted by Eq. 2, result in $\sigma_{0} \approx 3 \times 10^{-5} \mathrm{~S} / \mathrm{cm}$ (for $T=300 \mathrm{~K}$ ) and in $L_{0} / \gamma$ $\approx 1.5$, which is the ration between the minimum hopping distance and the charge carrier localization radius. The fitting was performed by considering a set of electronic states comprised in an energy interval $\Delta E=0.5 \mathrm{eV}$. This energetic interval is a good approximation for the distribution of electronic states in organic materials, which is well described by a Gaussian distribution with a disorder parameter of $0.1 \mathrm{eV}$ [26-29]. Additionally, it is possible to determine $L_{0}$ as $4.2 \mathrm{~nm}$, considering an electronic localization radius of

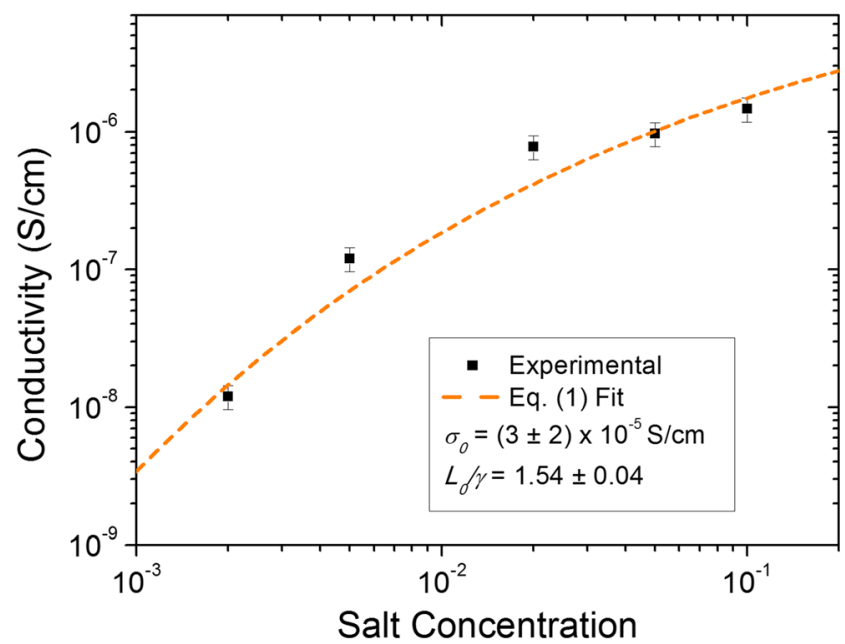

Fig. 4 Conductivity of the PLEC-doped active layer as a function of the dopant salt concentration

$2.8 \mathrm{~nm}$, as previously reported [16, 25]. This value of $L_{0}$ yields a density of electronic charge carrier of $1.310^{19} \mathrm{~cm}^{-3}$ for the extrinsic semiconductor.

The temperature dependence of the conductivity of the polymer (ADS_GE) ECDL is shown in Fig. 5a. The temperature range considered is above the PEO glass transition, where the ionic conductivity is high enough to provide the needed ionic charge separation to promote the electrochemical doping of the semiconducting polymer. This result shows that the conductivity of the electrochemically $p$-doped semiconductor is weakly dependent on the temperature, as expected for an extrinsic semiconductor. Figure 5 presents the temperature dependence of the Mott characteristic hopping temperature, $T_{\mathrm{h}}$, obtained from the differentiation of the $\ln (\sigma) \mathrm{vs} . T^{-1 / 4}$ plot, according to Eq. 2. This result shows that $T_{\mathrm{h}}$ has a sigmoidal dependence on temperature, leaning towards zero for increasing temperatures.

To explain the temperature dependence of the electrical conductivity of the polymeric blend, it is necessary to take into account few assumptions. First, that it is reasonable to suppose that the dissociated ionic charge is proportional to the PEO ionic conductivity, which is known to have Arrhenius dependence for temperatures above the glass transition temperature [30-32]. Another reasonable consideration is that the electronic charge carrier density in the active material is given by the sum of the intrinsic and extrinsic charge carrier densities and that the latter is proportional to the material ionic conductivity. Therefore, the density of electronic charge carriers in the organic semiconductor can be expressed by: $n(T)=n_{\mathrm{i}}+n_{\infty} \exp \left(-\frac{E_{i}}{K_{\mathrm{B}} T}\right)$, where $n_{\mathrm{i}}$ represents the concentration of intrinsic charge carriers, $E_{\mathrm{i}}$ is the activation energy of the ionic transport in the solid electrolyte, and $n_{\infty}$ a parameter related to the concentration of extrinsic charge carriers. Using the procedure above to obtain the Mott 

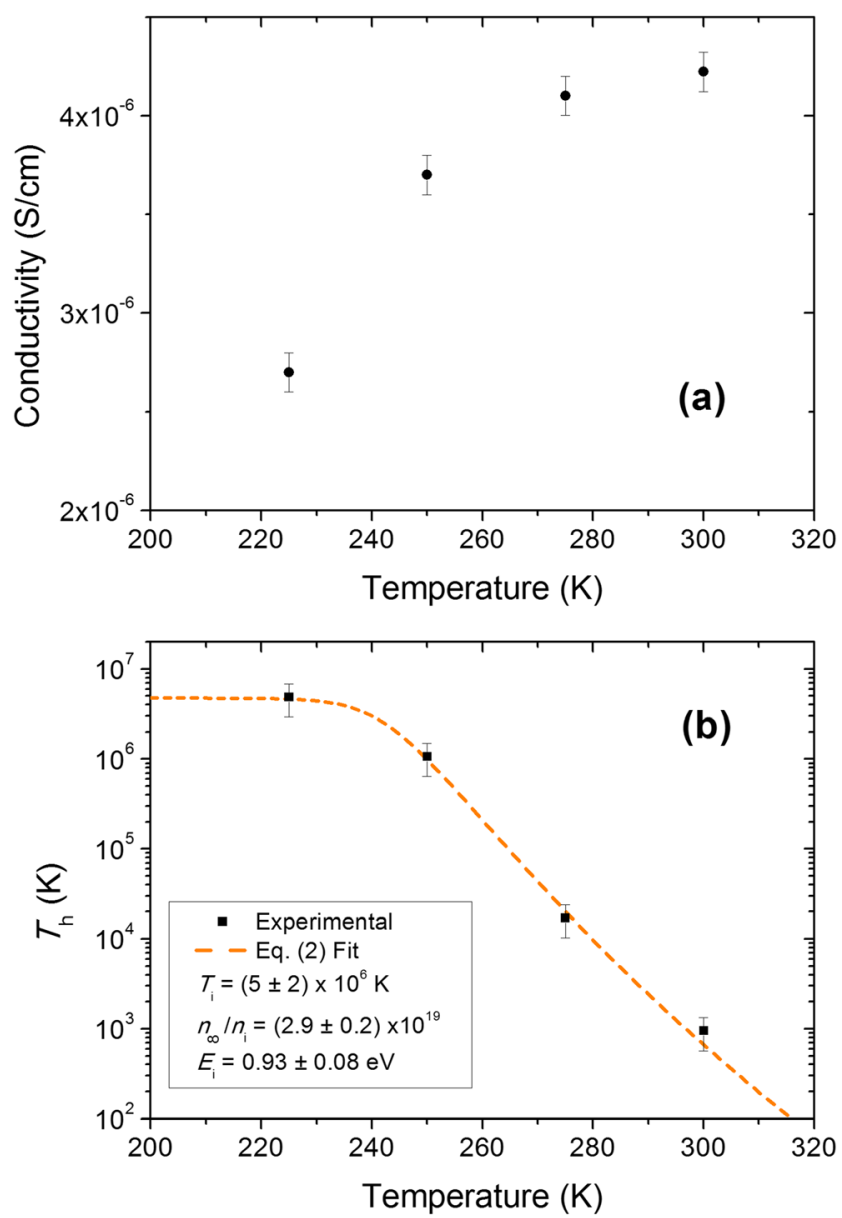

Fig. 5 a Conductivity of the PLEC-doped active layer as a function of the experimental temperature and $\mathbf{b}$ hopping temperature dependence on the experimental temperature

characteristic hopping temperature as a function of the charge carrier density (conductivity) from Eq. 2, and also considering the temperature dependence for the charge carrier density, it is possible to obtain the temperature dependence of the Mott characteristic hopping temperature:

$T_{\mathrm{h}}(T)=\frac{T_{i}}{1+\frac{n_{\infty}}{n_{i}} \exp \left(-\frac{E_{i}}{k_{\mathrm{B}} T}\right)}$

where $T_{\mathrm{i}}$ represents the Mott characteristic hopping temperature of the intrinsic semiconductor. The fitting of the experimental results using Eq. 3 is shown in Fig. 5b, resulting in the following parameters: $T_{\mathrm{i}} \approx 5 \times 10^{6} \mathrm{~K}, \frac{n_{\infty}}{n_{i}} \approx 2.9 \times 10^{19}$ and $E_{\mathrm{i}} \approx 0.93 \mathrm{eV}$. As expected, the activation energy obtained for the ionic charge transport is in accordance with previous results for solid electrolytes comprising PEO/LiTri [29-31]. The obtained value for the Mott characteristic hopping temperature of the intrinsic semiconductor $\left(T_{\mathrm{i}}=5 \times 10^{6} \mathrm{~K}\right)$ is consistent with the results obtained for several other semiconducting polymers in the literature [33-36]. This parameter is dependent on the fundamental parameters, as the density of states, the energy interval which comprises the density of states $\Delta E$, and the electronic charge carrier localization radius [35]. Considering the obtained value of $T_{\mathrm{i}}$, an electronic localization radius of $2.8 \mathrm{~nm}$ and $\Delta E=0.5 \mathrm{eV}$ for the intrinsic semiconductor, it was possible to determine the density of an intrinsic charge carrier of $5.410^{17} \mathrm{~cm}^{-3}$, in agreement with the typical density of electronic states in intrinsic organic semiconductors [24]. As expected, the concentration of intrinsic charge carriers is much lower than the maximum concentration of extrinsic charge carriers. Finally, in our representation of the density of charge carrier with the temperature, the relationship between the density of mobile ions in the polymer electrolyte, $n_{\mathrm{mi}}(T)$ and $n_{\infty}$ is given by $n_{\mathrm{mi}}(T)=n_{\infty} \exp \left(-\frac{E_{i}}{K_{\mathrm{B}} T}\right)$. Considering that, at room temperature, practically all salt molecules are dissociated in the polymer electrolyte, we can evaluate a concentration of mobile ions of $1.9 \times 10^{20} \mathrm{~cm}^{-3}$ for the blend composition of 1:1:0.1 (OS/PEO/LiTri). The temperature necessary to achieve this concentration of mobile ions from the obtained values for the $\frac{n_{\infty}}{n_{i}}$ ratio and from the density of intrinsic charge carriers was determined to be $(280 \pm 30) \mathrm{K}$, which is, considering the uncertain, close to room temperature.

\section{Conclusions}

The electrical properties of electrochemically doped polymeric semiconductors were studied by using PLECs operating at voltages above the device turn-on voltage, when the electrochemical doping regime is predominant. The experimental results show that the electric conductivity of the devices increases with the semiconductor doping level (associated with the Li salt concentration in the solid electrolyte), with the organic semiconductor concentration in the blend, and with the electronic charge carrier mobility. Additionally, the obtained results have shown that the electric conductivity for the whole device is weakly dependent on the experimental temperature, indicating that, for high-applied voltages, the PLEC apparent conductivity is approximately the conductivity of the completely doped organic semiconductor, as presumed in the phenomenological model (PM) used to describe the operation of PLECs. Moreover, we have shown that it is possible to obtain diverse parameters of the charge transport in the organic semiconductor by studying the electrical properties of PLECs in a high-voltage regime. In the current study were obtained properties of an polyfluorene derivative, as electronic localization radius of $2.8 \mathrm{~nm}$, minimum hopping distance of about $4.2 \mathrm{~nm}$, density of extrinsic charge carriers $1.310^{19} \mathrm{~cm}^{-3}$, density of intrinsic charge carriers as $5.410^{17} \mathrm{~cm}^{-3}$, and activation energy of the ionic charge transport about $0.93 \mathrm{eV}$ are in good agreement with expected values. 
Acknowledgments The authors acknowledge the financial support from National Institute of Organic Electronics, INEO (FAPESP grant \# 2008/57706-4) and FAPESP grant 2013/24461-7.

\section{References}

1. Pei Q, Yu G, Zhang C, Yang Y, Heeger AJ (1995) Science 269: 1086-1088

2. Hohertz D, Gao J (2008) Adv Mater 20:3298-3330

3. Tang S, Mindemark J, Araujo CMG, Brandell D, Edman L (2014) Chem Mater 26:5083-5088

4. Gao J, AlTal F (2014) Appl Phys Lett 104:143301

5. Yu Z, Li L, Gao H, Pei Q (2013) Sci China Chem 56(8):1075-1086

6. Asadpoordarvish A, Sandström A, Tang S, Granström J, Edman L (2012) Appl Phys Lett 100:193508 1-4

7. Yu Z, Wang M, Lei G, Liu J, Li L, Pei Q (2011) J Phys Chem Lett 2: 367-372

8. Tang S, Edman L (2010) J Phys Chem Lett 1:2727-2732

9. Matyba P, Yamaguchi H, Eda G, Chhowalla M, Edman L, Robinson ND (2010) ACS Nano 4(2):637-642

10. Matyba P, Yamaguchi H, Chhowalla M, Robinson ND, Edman L (2011) ACS Nano 5(1):574-580

11. Sandström A, Dam HF, Krebs FC, Edman L (2012) Nature. Communications 3(1002): 1-5

12. Sandström A, Asadpoordarvish A, Enevold J, Edman L (2014) Adv Mater 26:4975-4980

13. DeMello JC, Tesseler N, Graham SC, Friend RH (1998) Phys Rev B 57:12951-12963

14. Smith DL (1997) J Appl Phys 81:2869-2288

15. Pingree LSC, Rodovsky DB, Coffey DC, Bartholomew GP, Ginger DS (2007) J Am Chem Soc 129:15903-159010

16. Matyba P, Andersson MR, Edman L (2008) Org Electron 9:699710
17. van Reenen S, Matyba P, Dzwilewski A, Janssen RAJ, Edman L, Kemerink M (2010) J Am Chem Soc 132:13776-13781

18. van Reenen S, Matyba P, Dzwilewski A, Janssen RAJ, Edman L, Kemerink (2011) Adv Funct Mater 21:1795-1802

19. Gozzi G, Cagnani LD, Faria RM, Santos LF (2014) J Sol State Electrochem 18:3181-3190

20. Gozzi G, Santos LF, Faria RM (2012) Europhys Lett 100(18001): $1-4$

21. Gozzi G, Faria RM, Santos LF (2012) Appl Phys Lett 101:113305 $1-5$

22. Santos LF, Pereira CJ (2013) Rev Bras Ens Fis 35(2):2314 1-8

23. Arkhipov VI, Heremans P, Emelianova EV, Adriaenssens GJ, Bassler HB (2004) J Non-Cryst Solids 338-340:603-606

24. Nicolai HT, Kuik M, Wetzelaer GAH, de Boer B, Campbell C, Risko C, Brédas JL, Blom M (2012) Nat Mater 11:882-887

25. Mott NF (1969) Philos Mag 19:835

26. Ihnatsenka S, Crispin X, Zozoulenko IV (2015) Phys Rev B 92: 035201

27. Oelerich JO, Huemmer D, Baranovskii SD (2012) Phys Rev Lett 108:226403

28. van der Holst JJM, van Oost FWA, Coehoorn R, Bobbert PA (2011) Phys Rev B 83:085206

29. Schmechel R (2003) J Appl Phys 93(8):4653-4660

30. Gadjourova Z, Andreev YG, Tunstall DP, Bruce PG (2001) Lett Nat 412:520-523

31. Caruso T, Capolconi S, Cazzanelli E, Agostino RG, Villano P, Passerini S (2002) Ionics 8:36-43

32. Ramanujachary KV, Tong X, Lu Y, Kohn J, Greenblatt M (1996) J Appl Polym Sci 11:1449-1456

33. Aleshin AN, Sandberg H, Stubb H (2001) Synth Met 121:14491450

34. Nardes AM, Janssen RAJ, Kemerink M (2008) Adv Funct Mater 18:865-871

35. Maddison DS, Tansley TL (1992) J Appl Phys 72:4677-4682

36. Novak M, Kokanović I, Babić D, Baćani M, Tonejc A (2009) Synth Met 159:649-653 